\title{
荞麦地鼠尾草根中的二萜类成分
}

\author{
夏光惠 ${ }^{a}$ 李元平 $b$ 毕德文 ${ }^{b}$ 张兰军 $b$ \\ 李宏哲 $*, a$ 高林花 ${ }^{b}$ 王利勤 $*, b$ \\ $\left({ }^{a}\right.$ 云南中医学院中药学院 昆明 650500) \\ ( ${ }^{b}$ 云南师范大学化学化工学院 昆明 650500)
}

\begin{abstract}
摘要 对民间丹参代用品养麦地丹参(Salvia kiaometiensis Lévl.)乙醇提取物进行化学成分研究, 通过现代多种色谱技术 和波谱技术，从中分离并鉴定 16 个二萜类化合物，分别是 6,8,11,13-abietatetraen-12-ol dimer (1), kiaometin (2), miltirone (3), tanshinone IIA (4), crypotanshione (5), tanshindiol C (6), ferruginol (7), 2-isopropyl-8-methylphenanthrene-3,4-dione (8), tanshinone I (9), trijuganone B (10), dihydrotanshinone I (11), grandifolias C (12), przewalskin (13), tanshinlactone (14), 6,7,8,9-tetrahydro-1,6,6-trimethylfuro[3,2-c]naphtha[2,1-e]oxepine-10,12-dione (15), almilalbanone (16). 其中, 化合物 1 为 新的松香烷型二萜的二聚体, 2 为新的降松香烷型二萜化合物, 其 B 环 7,8 位开环后又通过醚桥连接起来. 所有化合物 均为首次从该种植物中得到.
\end{abstract}

关键词＼cjkstart菾麦地鼠尾草; 鼠尾草属; 唇形科; 松香烷型二萜

\section{Diterpenes from the Roots of Salvia kiaometiensis Lévl}

\author{
Xia, Guanghui ${ }^{a}$ \\ Li, Yuanping ${ }^{b}$ \\ Bi, Dewen ${ }^{b}$ \\ Zhang, Lanjun ${ }^{b}$ \\ Li, Hongzhe ${ }^{*, a}$ \\ Gao, Linhua ${ }^{b}$ \\ Wang, Liqin*,b \\ ( ${ }^{a}$ College of Pharmaceutical Science, Yunnan University of Traditional Chinese Medicine, Kunming 650050) \\ $\left({ }^{b}\right.$ Faculty of Chemistry and Engineering, Yunnan Normal University, Kunming 650050)
}

\begin{abstract}
Through a variety of chromatographic techniques and spectroscopic methods, sixteen diterpenes were isolated and identified from the ethanol extraction of the roots of Salvia kiaometiensis Lévl. Their structures were identified to be 6,8,11,13-abietatetraen-12-ol dimer (1), kiaometin (2), miltirone (3), tanshinone IIA (4), crypotanshione (5), tanshindiol C (6), ferruginol (7), 2-isopropyl-8-methylphenanthrene-3,4-dione (8), tanshinone I (9), trijuganone B (10), dihydrotanshinone I (11), grandifolias C (12), przewalskin (13), tanshinlactone (14), 6,7,8,9-tetrahydro-1,6,6-trimethylfuro[3,2-c]naphtha[2,1-e]oxepine10,12-dione (15), and salmilalbanone (16). Compound $\mathbf{1}$ is a new abietane diterpenes dimer, and compound $\mathbf{2}$ is a new 7,8:7,20-diepoxy-nor-abietane diterpenes. All of the diterpenes were isolated from this plant for the first time.

Keywords Salvia kiaometiensis; Salvia; Lamiaceae; abietane diterpenes
\end{abstract}

菾麦地鼠尾草(Salvia kiaometiensis Lévl.)为唇形科 (Lamiaceae) 鼠尾草属(Salvia L.)植物, 主产于云南东北 部和四川中南部 ${ }^{[1]}$. 本属中的多种植物具有极高的药用 价值, 是一类重要的药用植物资源. 《中华本草》记载 菾麦地鼠尾草具有活血调经、化瘀止痛之功, 主治月经 不调、痛经、经闭、产后瘀阴腹痛、肝脾肿大、关节痛、 胸绞痛、心悸、失眠等 ${ }^{[2]}$. 菾麦地鼠尾草在民间常代丹 参使用, 故又叫菾麦地丹参或土丹参, 其功效和主治与
丹参相似. 目前, 未见国内外对其进行相关研究的报道. 为了更好地利用鼠尾草属植物的药用资源，从民间医药 汲取养分, 我们对土丹参化学成分开展了研究, 从中分 离纯化得到 16 个二萜类化合物 (见图 1), 分别是 6,8,11,13-abietatetraen-12-ol dimer (1), kiaometin (2), miltirone $(\mathbf{3})^{[3]}$, tanshinone IIA $(\mathbf{4})^{[4]}$, crypotanshione $(\mathbf{5})^{[4]}$, tanshindiol $\mathrm{C}(\mathbf{6})^{[5]}$, ferruginol $(7)^{[6]}$, 2-isopropyl-8methylphenanthrene-3,4-dione $(\mathbf{8})^{[7]}$, tanshinone I $(\mathbf{9})^{[4]}$,

\footnotetext{
* Corresponding author. E-mail: kibwang@sohu.com

Received March 2, 2017; revised May 22, 2017; published online June 2, 2017.

Project supported by the National Natural Science Foundation of China (No. 31100143).

国家自然科学基金(No. 31100143)资助项目.
} 

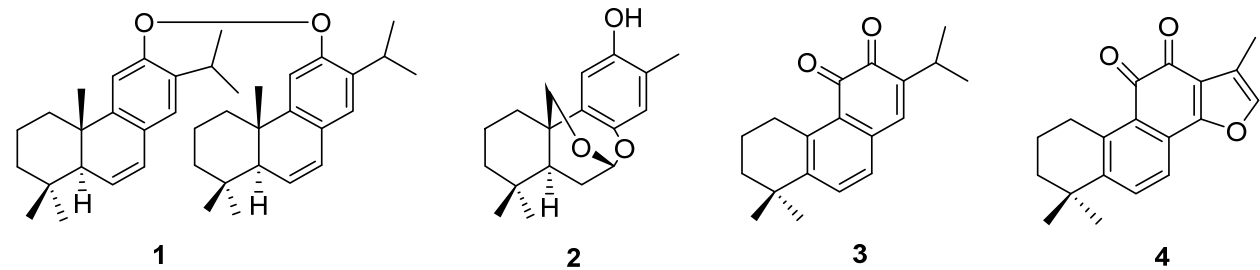<smiles>CC1COC2=C1C(=O)C(=O)c1c2ccc2c1CCCC2(C)C</smiles><smiles>Cc1coc2c1C(=O)C(=O)c1c-2ccc2c1CC[C@@H](O)[C@@H]2O</smiles><smiles>CC(C)c1cc2c(cc1O)[C@@]1(C)CCC[C@H](C)[C@H]1CC2</smiles><smiles>Cc1cccc2c3c(ccc12)C=C(C(C)C)C(=O)C3=O</smiles><smiles>Cc1coc2c1C(=O)C(=O)c1c-2ccc2c(C)cccc12</smiles><smiles></smiles><smiles>Cc1cccc2c3c(ccc12)C(=O)C(=O)C1=C3OCC1C</smiles>

11<smiles>Cc1cc2c(cc1O)[C@@]1(CO)CCC[C@H](C)[C@H]1CC2</smiles>

12<smiles></smiles>

13<smiles>Cc1cccc2c1ccc1c3occ(C)c3c(=O)oc21</smiles>

14<smiles>Cc1coc2c1c(=O)oc(=O)c1c3c(ccc12)C(C)(C)CCC3</smiles>

15<smiles>CC1=CCCc2c1ccc1c(=O)oc(=O)oc21</smiles>

16

图 1 化合物 1 16 的结构

Figure 1 Chemical structures of compounds $\mathbf{1} \sim \mathbf{1 6}$

trijuganone B $(\mathbf{1 0})^{[8]}$, dihydrotanshinone I $(\mathbf{1 1})^{[4]}$, grandifolia C (12 $)^{[9]}$, przewalskin $(\mathbf{1 3})^{[10]}$, tanshinlactone $(\mathbf{1 4})^{[4]}$, 6,7,8,9-tetrahydro-1,6,6-trimethylfuro[3,2-c]naphtha[2,1-e]oxepine-10,12-dione (15) $)^{[11]}$, salmilalbanone $(\mathbf{1 6})^{[5]}$. 其中, 化合物 1 为新的松香烷型二萜二聚体, 2 为新的降松香 烷型二萜, 其 B 环 7,8 位开环后又通过醚桥连接起来. 所有化合物均为首次从该植物中得到.

\section{1 结果与讨论}

化合物 1 为无色油状物质, 其 HR-EIMS 给出化合 物的分子式为 $\mathrm{C}_{40} \mathrm{H}_{54} \mathrm{O}_{2}$ (测量值 $m / z$ 566.4117, 计算值为 566.4124), 不饱和度为 14 ; 其 ${ }^{1} \mathrm{H}$ NMR 和 ${ }^{13} \mathrm{C} \mathrm{NMR}$ 显示 该化合物结构中有 20 个碳原子, 其中有 5 个为 $\mathrm{CH}_{3}$ 碳, 4 个为 $\mathrm{sp}^{2}$ 杂化的 $\mathrm{CH}$ 碳, 4 个为 $\mathrm{sp}^{2}$ 杂化的 $\mathrm{C}$. 该化合物的 核磁图谱数据与文献报道的 6,8,11,13-abietatetraen$12-\mathrm{ol}^{[12]}$ 的核磁图谱数据 (见表 1) 一致, 但分子式 $\mathrm{C}_{40} \mathrm{H}_{54} \mathrm{O}_{2}$ 中碳原子和氧原子个数及其不饱和度却为后 者的两倍, 而氢原子个数比后者两倍少两个氢原子, 所 以化合物 1 应为 6,8,11,13-abietatetraen-12-ol 的二聚体, 并且以过氧键相连. 综上所述, 该化合物结构鉴定为 6,8,11,13-abietatetraen-12-ol dimer.

化合物 2 为棕色油状液体, 其 HR-EIMS 给出化合 物的分子式为 $\mathrm{C}_{18} \mathrm{H}_{24} \mathrm{O}_{3}$ (测量值 $m / z$ 288.1718, 计算值为 $288.1725)$, 不饱和度为 7 ; 其 ${ }^{1} \mathrm{H}$ NMR 和 ${ }^{13} \mathrm{C} \mathrm{NMR}$ 显示
该化合物结构中有 18 个碳原子, 其中 3 个为 $\mathrm{CH}_{3}$ 碳, 5 个为 $\mathrm{CH}_{2}$ 碳 (1 个为连氧原子的 $\mathrm{CH}_{2}$ 碳), 4 个 $\mathrm{CH}$ 碳(化学 位移为 95.6 的 $\mathrm{CH}$ 可能为半缩醛 $\mathrm{CH}, 2$ 个为苯环上的 $\mathrm{CH}), 6$ 个 $\mathrm{C}$ (4 个为苯环上的 $\mathrm{C}$, 苯环上这四个 $\mathrm{C}$ 中化学 位移为 148.1 和 148.7 的两个 $\mathrm{C}$ 可能连接了氧原子). 该 化合物可能为降二萜化合物. 其所含碳原子个数及碳原 子的级别与已知化合物 13 (przewalskin)非常相似, 最大 的区别在于化合物 2 中连氧原子的 $\mathrm{CH}$ 碳化学位移为 $\delta$ 95.6, 而化合物 13 中连氧原子的 $\mathrm{CH}$ 碳化学位移为 $\delta$ 70.2 , 两者苯环上的碳化学位移也稍有不同. 化合物 2 的 HMQC 图谱显示为 $\delta_{\mathrm{C}} 95.6$ 上所连的氢为 $\delta 5.53$ 的氢, $\mathrm{HMBC}$ 图谱显示该氢与 $\delta_{\mathrm{C}} 47.7$ (C-5), 27.7 (C-6), 148.7 (C-8), 70.7 (C-20)相关; HMBC 图谱显示连氧原子的亚 甲基碳 $\left(\delta_{\mathrm{C}} 70.7\right)$ 上的氢 $\left(\delta_{\mathrm{H}} 4.21,4.04\right)$ 与 $\delta_{\mathrm{C}} 31.2(\mathrm{C}-1)$, 37.8 (C-10), 47.7 (C-5), 95.6 (C-7), 135.7 (C-9)相关. 前 述 $\mathrm{HMBC}$ 相关和波谱特征说明 $\delta 95.6$ 的碳原子为 7 位 碳，它与 20 位碳原子和 8 位碳原子之间分别通过氧原子 连接成两个醚桥. NOESY 图谱中的 H-20 ( $\left.\delta_{\mathrm{H}} 4.21\right)$ 与 $\mathrm{H}-19\left(\delta_{\mathrm{H}} 1.06\right), \mathrm{H}_{\beta}-2\left(\delta_{\mathrm{H}} 1.77\right)$ 相关, 说明 20 位 $\mathrm{CH}_{2}$ 与 7 位碳原子间形成的醚桥为 $\beta$ 位. 综上所述，化合物 2 的 结构鉴定为 16,17-nor-7,8:7 $\beta, 20$-diepoxy-13-methyl-abieta-8,11,13-tien12-ol (2), 将其命名为 kiaometin. B 环 7,8 位开环后又通过醚桥连接起来的降松香烷型二萜为首 次从鼠尾草属植物中得到. 
表 1 化合物 1 和 2 的 ${ }^{1} \mathrm{H} \mathrm{NMR}$ 和 ${ }^{13} \mathrm{C}$ NMR 数据(500/125 MHz, $\mathrm{CDCl}_{3}$ )

Table $1{ }^{1} \mathrm{H}$ and ${ }^{13} \mathrm{C}$ NMR $\left(500 / 125 \mathrm{MHz}, \mathrm{CDCl}_{3}\right)$ data of compounds $\mathbf{1}$ and 2

\begin{tabular}{|c|c|c|c|c|c|}
\hline 1 & $\delta_{\mathrm{C}}$ & $\delta_{\mathrm{H}}(J / \mathrm{Hz})$ & 2 & $\delta_{\mathrm{C}}$ & $\delta_{\mathrm{H}}(J / \mathrm{Hz})$ \\
\hline $1,1^{\prime}$ & $36.2 \mathrm{t}$ & $1.65(\mathrm{dd}, 13.0,3.7), 2.19$ & 1 & $31.2 \mathrm{t}$ & $1.91(\mathrm{td}, 13.0,4.4), 1.46(\mathrm{~d}, 13.0)$ \\
\hline $2,2^{\prime}$ & $19.0 \mathrm{t}$ & $1.78(\mathrm{tt}, 8.3,3.2), 1.69(\mathrm{tt}, 10.6,3.5)$ & 2 & $18.7 \mathrm{t}$ & $1.67(\mathrm{~m}), 1.77(\mathrm{dd}, 13.4,13.4)$ \\
\hline $3,3^{\prime}$ & $41.1 \mathrm{t}$ & $1.53(\mathrm{~d}, 15.8), 1.27(\mathrm{~d}, 6.9)$ & 3 & $40.6 \mathrm{t}$ & $1.20(\mathrm{dt}, 3.4,13.3), 1.55(\mathrm{~d}, 13.3)$ \\
\hline $4,4^{\prime}$ & $32.6 \mathrm{~s}$ & & 4 & $34.4 \mathrm{~s}$ & \\
\hline $5,5^{\prime}$ & $51.5 \mathrm{~d}$ & $2.10(\mathrm{~d}, 2.8)$ & 5 & $47.7 \mathrm{~d}$ & $1.66(\mathrm{~m})$ \\
\hline $6,6^{\prime}$ & $130.3 \mathrm{~d}$ & $5.98(\mathrm{dd}, 9.6,2.6)$ & 6 & $27.7 \mathrm{t}$ & $2.08(\mathrm{~d}, 6.9), 1.66(\mathrm{~m})$ \\
\hline $7,7^{\prime}$ & $126.9 \mathrm{~d}$ & $6.42(\mathrm{dd}, 9.6,2.9)$ & 7 & $95.6 \mathrm{~d}$ & $5.53(\mathrm{~d}, 3.0)$ \\
\hline $8,8^{\prime}$ & $131.5 \mathrm{~s}$ & & 8 & $148.7 \mathrm{~s}$ & \\
\hline $9,9^{\prime}$ & $141.1 \mathrm{~s}$ & & 9 & $135.7 \mathrm{~s}$ & \\
\hline $10,10^{\prime}$ & $37.6 \mathrm{~s}$ & & 10 & $37.8 \mathrm{~s}$ & \\
\hline $11,11^{\prime}$ & $113.1 \mathrm{~d}$ & $6.46(\mathrm{~s})$ & 11 & $120.8 \mathrm{~d}$ & $6.69(\mathrm{~s})$ \\
\hline $12,12^{\prime}$ & $150.5 \mathrm{~s}$ & & 12 & $148.1 \mathrm{~s}$ & \\
\hline $13,13^{\prime}$ & $133.1 \mathrm{~s}$ & & 13 & $122.8 \mathrm{~s}$ & \\
\hline $14,14^{\prime}$ & $119.9 \mathrm{~d}$ & $6.99(\mathrm{~s})$ & 14 & $110.8 \mathrm{~d}$ & $6.69(\mathrm{~s})$ \\
\hline $15,15^{\prime}$ & $27.4 \mathrm{~d}$ & 3.21 (七重峰) & 15 & $15.2 \mathrm{q}$ & $2.20(\mathrm{~s})$ \\
\hline $16,16^{\prime}$ & $22.8 \mathrm{q}$ & $1.25(\mathrm{~d}, 6.9)$ & & & \\
\hline $17,17^{\prime}$ & $22.6 \mathrm{q}$ & $1.24(\mathrm{~d}, 6.9)$ & & & \\
\hline $18,18^{\prime}$ & $20.6 \mathrm{q}$ & $1.02(\mathrm{~s})$ & 18 & $31.9 \mathrm{q}$ & $0.84(\mathrm{~s})$ \\
\hline $19,19^{\prime}$ & $22.5 \mathrm{q}$ & $1.03(\mathrm{~s})$ & 19 & $22.5 \mathrm{q}$ & $1.06(\mathrm{~s})$ \\
\hline $20,20^{\prime}$ & $32.8 \mathrm{q}$ & $0.96(\mathrm{~s})$ & 20 & $70.7 \mathrm{t}$ & $4.20(\mathrm{~d}, 10.8), 4.03(\mathrm{~d}, 10.8)$ \\
\hline
\end{tabular}

\section{2 结论}

通过多种色谱技术和波谱技术, 对土丹参根化学成 分进行分离和结构鉴定, 从其乙醇提取物中分离得到二 萜类化合物 16 个, 均为鼠尾草属植物中常见的结构类 型, 所有化合物都为首次从该植物中得到. 所得化合物 4 (tanshinone IIA, 丹参酮 IIA)、5 (crypotanshione, 隐丹 参酮)和 7 (ferruginol, 弥罗松酚) 在该植物中含量较大, 其中丹参酮 IIA 为丹参治疗心血管等疾病的主要有效成 分, 也是历版药典含量测定项检测物质, 而隐丹参酮是 丹参抗菌的主要有效成分. 除了这些含量较大的有效成 分外, 其他量较小的成分也多同丹参的有效成分, 这从 化学角度对土丹参有丹参的类似功效提供了证据, 对开 发新的药用资源意义深远.

\section{3 实验部分}

\section{1 仪器与试剂}

质谱用 Waters Autospec Premier 776 质谱仪测定; 核磁共振谱由 Bruker DRX 500MHz 和 Avance $600 \mathrm{MHz}$ 核磁共振仪测定, TMS 为内标; 柱层析硅胶(100-200 目 与 $200 \sim 300$ 目)和硅胶薄层板(GF254)购至青岛谱科分 离材料有限公司; Sephadex LH-20, 瑞士 GE Healthcare 公司; MCI 反相材料 $(75 \sim 150 \mathrm{~mm})$ 为日本 Mitsubishi Chemical corporation 生产. 所用有机溶剂皆为工业级, 经蒸馏后使用.

\section{2 植物材料}

植物材料为荞麦地鼠尾草的根, 2014 年 10 月采自 云南昭通巧家，经作者李宏哲(云南中医学院副教授)鉴 定为唇形科鼠尾草属植物菾麦地鼠尾草(Salvia kiaometiensis Levl.), 标本藏于云南中医学院博物馆腊叶标 本馆, 凭证标本号 2014008.

\section{3 提取与分离}

称取干燥的土丹参药材 $(16.0 \mathrm{~kg}$ )粉碎后用 $86 \%$ 乙醇 室温提取 5 次, 减压蒸馏浓缩得浸膏 $1332 \mathrm{~g}$, 用蒸馏水 溶解浸亳后加入等量的乙酸乙酯萃取 3 次得乙酸乙酯萃 取物 $480 \mathrm{~g}$, 该萃取物 $480 \mathrm{~g}$ 经硅胶 (100 200 目)柱色谱, 以石油醚-乙酸乙酯 $(V: V=20: 1,10: 1,7: 1,5: 1$, $3: 1,1: 1,1: 3,1: 5$ )梯度洗脱, 经薄层色谱(TLC)检 测合并得 10 个组分 $\mathrm{A} \sim \mathrm{J}$.

B (11g)部分经反复硅胶柱色谱[石油醚-乙酸乙酯 $(V: V=15: 1)$, 氯仿-甲醇 $(V: V=300 ： 1)$, 石油醚-氯 仿 $(V: V=1: 1)$ ]得到化合物 $7(2.5 \mathrm{~g})$.

C $(21.5 \mathrm{~g})$ 部分放置过程中析出大量红色粉末, 为 化合物 4 (15 g), 母液经硅胶柱色谱[石油醚-乙酸乙酯 $(V: V=25: 1)]$ 分为 4 个组分, $\mathrm{C} 1$ 经反复硅胶柱色谱[石 油醚-乙酸乙酯 $(V: V=40: 1)$ ], [石油醚-氯仿 $(V: V=$ $1: 1)$, 氯仿-甲醇 $(V: V=150: 1)]$ 后得到化合物 1 (81 $\mathrm{mg})$ 和 $4(83 \mathrm{mg})$. C2 经 Sephadex LH-20 和硅胶柱色谱 [石油醚-乙酸乙酯 $(V: V=20: 1)$ ] 得到化合物 3 (940 $\mathrm{mg}) . \mathrm{C} 4$ 经硅胶柱色谱[石油醚-乙酸乙酯 $(V: V=40$ : 
1)]层析, 洗脱液放置析出晶体, 重结晶得化合物 $\mathbf{1 4}$ (200 mg).

D $(21 \mathrm{~g})$ 部分经硅胶柱色谱[石油醚-乙酸乙酯 $(V$ : $V=11 ： 1)]$ 分为 3 个组分, D3 经 Sephadex LH-20 和硅胶 柱色谱[石油醚-乙酸乙酯 $(V: V=20: 1)$, 石油醚-丙酮 $(V: V=20: 1)]$ 得化合物 $9(169 \mathrm{mg})$.

F $(51 \mathrm{~g})$ 部分经硅胶柱色谱[石油醚-乙酸乙酯 $(V$ : $V=13: 1 \sim 1: 1)$ ]划分为 4 个组分, $\mathrm{F} 1$ 经硅胶柱色谱[石 油醚-乙酸乙酯 $(V: V=35: 1)]$ 分为 4 个组分, $\mathrm{F} 1 \mathrm{c}$ 经 Sephadex LH-20 得到化合物 $15(112 \mathrm{mg})$. F1d 放置析出 晶体, 重结晶得到化合物 $\mathbf{1 6}(201 \mathrm{mg})$, 母液经硅胶柱色 谱[石油醚-乙酸乙酯 $(V: V=30: 1)$ ] 得化合物 $1(6 \mathrm{mg})$. F3 经反复硅胶柱色谱[石油醚-乙酸乙酯 $(V: V=30: 1)$, 氯仿一丙酮 $(V: V=50: 1)]$, Sephadex LH-20得化合物 13 $(79 \mathrm{mg}), 2(120 \mathrm{mg})$. F4 经硅胶柱色谱[石油醚-乙酸乙酯 $(V: V=20: 1)$ ]得到 3 个组分, $\mathrm{F} 4 \mathrm{c}$ 经硅胶柱色谱[石油 醚-乙酸乙酯 $(V: V=20: 1)]$ 得到 3 个组分, 第二份为 $24.7 \mathrm{~g}$, 取 $3.3 \mathrm{~g}$ 经硅胶柱色谱[石油醚一乙酸乙酯 $(V$ : $V=10: 1)$ ]得化合物 $5(1.15 \mathrm{~g})$. 第三份经硅胶柱色谱 [石油醚-乙酸乙酯 $(V: V=10: 1)$ ], MCI 柱层析[甲醇水 $(V: V=6: 4,7: 3,8: 2,10: 0)]$ 得 10 个组分, 第 5 份经硅胶柱色谱[石油醚-乙酸乙酯 $(V: V=10 ： 1)$ ]得化 合物 $12(361 \mathrm{mg})$. 第 8 份经 MCI 柱层析[甲醇-水 $(V$ : $V=6: 4,7: 3,8: 2,10: 0)$ ]得两个组分, 分别经硅胶 柱色谱[石油醚-乙酸乙酯 $(V: V=12: 1)$ ] 得化合物 $\mathbf{1 0}$ (11 mg), 11 (5 mg).

$\mathrm{K}(77 \mathrm{~g})$ 部分经硅胶柱色谱[氯仿一甲醇 $(V: V=35$ ： $1 \sim 0: 1)$ ]划段分为 10 个组分, $K 4$ 经反复硅胶柱色谱[氯 仿一甲醇 $(V: V=40: 1 \sim 20: 1)$ ], MCI 反相柱色谱[甲醇 -水 $(V: V=6: 4,7: 3,8: 2,10: 0)$ ]得化合物 $6(54.2$ $\mathrm{mg})$.

6,8,11,13-Abietatetraen-12-ol dimer (1): $[\alpha]_{\mathrm{D}}^{20.6}-$ 125.83 (c $\left.0.160, \mathrm{CHCl}_{3}\right)$; UV $(\mathrm{MeOH}) \lambda_{\max }[\log \varepsilon /(\mathrm{L} \cdot$ $\left.\mathrm{mol}^{-1} \cdot \mathrm{cm}^{-1}\right)$ ]: $239(3.51) \mathrm{nm} ;{ }^{1} \mathrm{H}$ NMR and ${ }^{13} \mathrm{C}$ NMR see Table 1; IR (KBr) $v_{\max }$ : 2958, 1764, 1618, 1461, 1423, $1156 \mathrm{~cm}^{-1}$; HR-EI-MS calcd for $\mathrm{C}_{40} \mathrm{H}_{54} \mathrm{O}_{2} 566.4124$, found 566.4117 .

16,17-Nor-7,8:7 $\beta, 20$-diepoxy-13-methyl-abieta-8,11, 13-tien-12-ol (2): $[\alpha]_{\mathrm{D}}^{20.6}-155.33\left(c 0.150, \mathrm{CHCl}_{3}\right)$; UV

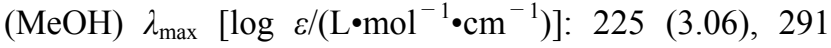
(2.71) nm; ${ }^{1} \mathrm{H}$ NMR and ${ }^{13} \mathrm{C}$ NMR see Table 1; IR (KBr) $v_{\max }: 3413,2950,2873,1498,1458,1411,1181,1030$ $\mathrm{cm}^{-1}$; HR-EI-MS calcd for $\mathrm{C}_{18} \mathrm{H}_{24} \mathrm{O}_{3} 288.1725$, found 288.1718 .

辅助材料 (Supporting Information) 新化合物的 $1 \mathrm{D} / 2 \mathrm{D} \mathrm{NMR}$ 、高分辨质谱等谱图. 这些材料可以免费从 本刊网站(http://sioc-journal.cn/)上下载.

\section{References}

[1] Delectis Flora Reipublicae Popularis Sinicae Edita Flora Reipublicae Popularis Sinicae, Vol. 66, Science Press, Beijing, 1977, pp. $129 \sim 130$ (in Chinese).

(中国科学院中国植物志编辑委员会编, 中国植物志, 第 66 卷, 科学出版社, 北京, 1977, pp. 129 130.)

[2] Editorial Board of Chinese Materia Medica Chinese Materia Medica, Vol. 19, Shanghai Science and Technology Press, Shanghai, 1999, p. 168 (in Chinese).

(国家中医药管理局《中华本草》编委会，中华本草，第 19 卷，上 海科学技术出版社, 上海, 1999, p. 168.)

[3] Cao, C. Q.; Sun, L. R.; Lou, H. X.; Ji, M. Lishizhen Med. Mater. Med. Res. 2009, 20, 636 (in Chinese). (曹春泉, 孙隆儒, 娄红祥, 季梅, 时珍国医国药, 2009, 20, 636.)

[4] Zhu, L. P.; Xiang, C.; Zhuang, W. T.; He, J.; Li, P.; Li, B. C. Nat. Prod. Res. Dev. 2013, 25, 785 (in Chinese).

(朱路平, 向诚, 庄文婷, 何静, 李鹏, 李宝才, 天然产物研究与 开发, 2013, 25, 785.)

[5] Cao, C. Q.; Sun, L. R.; Wang, X. N. Chin. Trad. Herb. Drugs 2009, 40, 173 (in Chinese).

(曹春泉, 孙隆儒, 王小宁, 中草药, 2009, 40, 173.)

[6] Hasegawa, S.; Hirose, Y. Phytochemistry 1982, 21, 643.

[7] Onitsuka, M.; Fujiu, M.; Shinma, N.; Maruyama H. B. Chem. Pharm. Bull. 1983, 31, 1670 .

[8] Lan, T. F.; Yu, Z. Y.; Wang, D. J.; Wang, X.; Guan, R. J. Chin. Trad. Herb. Drugs 2011, 42, 466 (in Chinese). (蓝天凤，于宗渊，王岱杰，王晓，管仁军，中草药， 2011，42， 466.)

[9] Kang, J.; Li L.; Wang, D. D.; Wang, H. Q.; Liu, C.; Li, B. M.; Yan, Y.; Fang, L. H.; Du, G. H.; Chen, R. Y. Phytochemistry 2015, 116, 337.

[10] Li, B.; Niu, F. D.; Lin, Z. W.; Zhang, H. J.; Wang, D. Z.; Sun, H. D. Phytochemistry 1991, 30, 3815.

[11] Mak, T. C. W.; Wong, N. C.; Chang, H. M.; Choang, T. F.; Chui, K. Y.; Hon, P. M.; Lee, C. M. J. Chem. Res. 1990, 877.

[12] Guillen, M. D.; Manzanos, M. J. Agric. Food Chem. 1999, 47, 3016.

[13] Han, G. H.; Li, Z. L.; Sun, L.; Hua, H. L. J. Shengyang Pharm. Univ. 2009, 26, 896 (in Chinese) (韩国华, 李占林, 孙琳, 华会明, 沈阳药科大学学报, 2009, 26, 896.)

(Li, L.; Fan, Y.) 